\title{
Optimal Control with an Isoperimetric Constraint Applied to Cancer Immunotherapy
}

\author{
Amine Hamdache \\ Faculty of Sciences Ben M'Sik, \\ P.O Box 7955 Sidi Othman, \\ Casablanca, Morocco
}

\author{
Ilias Elmouki \\ Faculty of Sciences Ben M'Sik, \\ P.O Box 7955 Sidi Othman, \\ Casablanca, Morocco
}

\author{
Smahane Saadi \\ Faculty of Sciences Ben M'Sik, \\ P.O Box 7955 Sidi Othman, \\ Casablanca, Morocco
}

\begin{abstract}
In this paper, a therapeutic strategy for the treatment of cancer using immunotherapy that aims to maximize the active immune response and to minimize the tumor cells level while reducing drugs side effects and treatment cost is proposed. Assume that the treatment amount that can be administered to a potential patient during therapy period is known precisely, an ODE model with control acting as an immunotherapy agent is presented and an optimal control problem is formulated to include an isoperimetric constraint on the immunotherapy treatment. The Pontryagin's maximum principle is used to characterize the optimal control taking into account the fixed isoperimetric constraint. The optimality system is derived and solved numerically using an adapted iterative method with a Runge-Kutta fourth order scheme and secant method routine.
\end{abstract}

\section{General Terms}

Cancer immunotherapy, Optimal control theory, Forward backward sweep method.

\section{Keywords}

Interleukin-2, Isoperimetric constraint, Pontryagin's maximum principle, Runge-Kutta fourth order scheme, Secant method routine

\section{INTRODUCTION}

Cancer is a general term applied to a large group of diseases that can affect any part of the body. One of its characteristics is the rapid proliferation of abnormal cells that can spread to other organs, forming what is called metastasis. Many cancers can be prevented by avoiding key risk factors. A significant number of cancers can be treated by surgery, immunotherapy and chemotherapy especially if detected early enough. In recent decades, mathematical modeling and optimal control theory are used as mathematical tools by scientists of biomathematics in the areas of oncology and epidemiology trying to better understand the evolution and stability of the disease for anticipating the action of the appropriate treatment and for finding the best way to administer the proper medications while minimizing side effects. It is noted with interest that many mathematical models were interested to the evolution and the treatment of some types of cancer such as brain cancer (Swanson and al. 2003 [1]), bladder cancer (Bunimovich and al. 2007 [2]) and prostate cancer (Celestia and al. 2009 [3]). Immunotherapy stimulates the natural defenses of the body commonly called immune response cells. However, the immunotherapy includes very different therapeutic strategies depending on whether they mobilize or enhance the resources of the patient's immune system (active immunotherapy) or on the contrary, they are using immunological reagents brought from outside (passive immunotherapy). Malignant tumors in humans are poorly immunogenic and they are not generally recognized as foreign by the body. Therefore, the defense mechanisms of the body must be stimulated with different methods such as cytokines. The Interleukin-2 is a substance normally secreted by our cells, it stimulates certain immune cells and it can also be synthesized by the genetic engineering. It is used in infusion or subcutaneously. The Interleukin-2 is utilized both in cancer treatment and in HIV treatment. Many scientific studies concerned with immunotherapy have proposed cellular and molecular immunology to develop therapeutic strategies that augment the antitumor responses [4]. Bunimovich and al. have developed a mathematical model describing dynamical behavior of tumor cells with immune system after injection of BCG immunotherapy (Bacillus Calmette-Guerin) in the human bladder [2]. De Pillis and al. have extended a mathematical model that governs cancer growth with chemotherapy treatments to include immunotherapy using the Interleukin-2 with tolerated doses [5]. Castiglione and al. have constructed a mathematical model of the immune-cancer interaction to study the effect of immunotherapy via dendritic cell vaccines using an optimal scheduling and a vaccine administration with impulsive controls [6]. Mathematical models proposed by Kirschner and al. [7] and Chan and al. [8] have been conducted to study a method of treating cancer by immunotherapy using the Interleukin- 2 in conjunction with the Adoptive Cellular Immunotherapy (ACI) that is a cell transfer in the host in order to transplant immune cells like 'NK' cells and 'CTL' cells in the single tumor-site. This type of immunotherapy consists indeed to administer to the patients an artificial Interleukin-2 cells produced in vitro. It is an important part of what is now called targeted therapies. In this work, a cancer model originally discussed in Kirschner and Panetta [7] is analyzed. This paper is organized as follows: Section 2 describes mathematical models of cancer immunotherapy with a control term, the first part presents the basic mathematical model of cancer treatment using the ACI immunotherapy and the second one focuses on the introduction of an isoperimetric constraint to the basic model. The analysis of the optimization problem is also presented in the same section. In section 3 , the iterative method is introduced and the numerical simulations are discussed. Finally, the results of this therapeutic approach are compared in the conclusion in section 4 . 


\section{MATHEMATICAL MODELS}

\subsection{Basic mathematical model of cancer treatment using immunotherapy}

In this section, an ODE system modeling the immune system dynamics of a cancer patient after the introduction of a treatment using immunotherapy (1) is presented. Three compartments characterizing the different populations are defined as follows: $x(t)$ the activated immune cells (effector cells), $y(t)$ the tumor cells and $z(t)$ the concentration of IL-2 cells in the single tumor-site compartment. Therefore, the model characterizing the interaction between these intervening cells is:

$$
\begin{aligned}
& \frac{d x}{d t}=c y-\mu_{2} x+\frac{p_{1} x z}{g_{1}+z}+u(t), \\
& \frac{d y}{d t}=r_{2} y(1-b y)-\frac{a x y}{g_{2}+y}, \\
& \frac{d z}{d t}=\frac{p_{2} x y}{g_{3}+y}-\mu_{3} y .
\end{aligned}
$$

In addition, assume that the parameters are all considered positive constants and the normalized initial data [9] for system (1) satisfy:

$$
\mathrm{x}_{0}=1 \geq 0, \mathrm{y}_{0}=1 \geq 0, \mathrm{z}_{0}=1 \geq 0
$$

The description of the different above model parameters (1) is listed in the following table:

Table 1. The description of parameters and terms used

\begin{tabular}{|c|c|}
\hline Parameters & Descriptions \\
\hline $\mathrm{c}$ & Tumor antigenicity rate \\
\hline$\mu_{2}$ & Natural mortality rate of effector cells \\
\hline $\mathrm{p}_{1}$ & Effector cells stimulation rate by IL-2 cells \\
\hline $\mathrm{g}_{1}$ & Half saturation for proliferation term \\
\hline $\mathrm{r}_{2}$ & Tumor cells growth rate \\
\hline $\mathrm{b}$ & Tumor cells volume of change \\
\hline $\mathrm{a}$ & Tumor cells loss rate \\
\hline $\mathrm{g}_{2}$ & Half-saturation for cancer clearance \\
\hline $\mathrm{p}_{2}$ & Self-limiting production rate of IL-2 cells \\
\hline $\mathrm{g}_{3}$ & Half-saturation of production \\
\hline$\mu_{3}$ & Natural mortality rate of IL-2 cells \\
\hline
\end{tabular}

The terms $\frac{\mathrm{p}_{1} \mathrm{xz}}{\mathrm{g}_{1}+\mathrm{z}}, \frac{\mathrm{axy}}{\mathrm{g}_{2}+\mathrm{y}}$ and $\frac{\mathrm{p}_{2} \mathrm{xy}}{\mathrm{g}_{3}+\mathrm{y}}$ are represented by Michaelis Menten form to indicate respectively: The saturated effects of the immune response, the tumor cells loss and the IL-2 source produced by effector cells. However, the term $r_{2} y(1-b y)$ represents the tumor logistic growth function. Note that the following parameters: $a, b, p_{1}, p_{2}, g_{2}$ and $g_{3}$ are derived from scientific experiments [7]. The control $u(t)$ represents an external source of effector cells using the Tumor Infiltrating Lymphocyte Therapy (TIL) to incubate effector cells derived from a tumor with a high concentration of external immune cells in vitro and then injected back into the patient at the tumor site using the Adoptive Cellular Immunotherapy (ACI) [7]. Experimentally, the possible values of $u(t)$ are between 0 and $\lambda=1000$ units per day during the 350 days of treatment period [7].

\subsection{Mathematical model of cancer immunotherapy with an isoperimetric constraint}

\subsubsection{Presentation of the model}

The basic model (1) is extended for considering the situation in which it is supposed that the precise amount of treatment that could be administered to the patient during the treatment period of 350 days is known. Then, an integral constraint is defined as follows:

$$
C=\int_{0}^{t_{\mathrm{f}}} u(t) \mathrm{dt},
$$

This type of constraint is known as an isoperimetric constraint [10]. For proceeding to establish suitable solution methods to this kind of problem, it must be converted to a more familiar form in order to use properly the Pontryagin's maximum principle. Thus, this problem can be handled by creating another state variable, $h(t)$, such that:

$$
\begin{aligned}
& \mathrm{h}^{\prime}(\mathrm{t})=u(\mathrm{t}), \\
& \mathrm{h}(0)=0, \\
& \mathrm{~h}\left(\mathrm{t}_{\mathrm{f}}\right)=\mathrm{C} .
\end{aligned}
$$

After the introduction of an isoperimetric constraint, the basic model (1) of cancer treatment using immunotherapy is presented by the following new system:

$$
\begin{aligned}
& \frac{d x}{d t}=c y-\mu_{2} x+\frac{p_{1} x z}{g_{1}+z}+u(t), \\
& \frac{d y}{d t}=r_{2} y(1-b y)-\frac{a x y}{g_{2}+y}, \\
& \frac{d z}{d t}=\frac{p_{2} x y}{g_{3}+y}-\mu_{3} y, \\
& \frac{d h}{d t}=u(t) .
\end{aligned}
$$

Our goal is obviously to maximize the benefits based on the immunotherapy effects while minimizing the systemic 'cost' of the control. Precisely, the principal objective is to be able to maximize the levels of effector cells as well as the Interleukin-2 cells and to reduce the tumor cells level while minimizing the 'cost' of treatment also including side effects of the drugs. Our control is a function $u(t)$ taking values between 0 and $\lambda=1000$ units. Note that, a drug amount of 1000 units per day is the maximum dosage available and zero is the minimum.

\subsubsection{The optimal control problem}

The problem is to maximize the objective function:

$J(u)=\int_{0}^{t_{f}} \frac{1}{2}\left(x^{2}(t)-y^{2}(t)+z^{2}(t)-W u^{2}(t)\right) d t$

where the positive parameter $\mathrm{W} \geq 0$ balances the size of the terms and it represents a weight factor characterizing a patient's level of acceptance of the treatment.

Based on the objectives of our optimal control problem, an objective function is constructed in a manner to maintain the 
same order of potency $(\mathrm{k}=2)$ for all terms within the integrand to ensure more consistency to our optimal control problem.

Mathematically, an optimal control $u^{*} \in \mathrm{U}$ is sought such that:

$$
J\left(u^{*}\right)=\max _{u \in \mathrm{U}} J(u)
$$

where $\mathrm{U}$ is the control set defined by:

$\mathrm{U}=\left\{u\right.$ Lebesgue - measurable, $\left.0 \leq u(\mathrm{t}) \leq \lambda, \mathrm{t} \in\left[0, \mathrm{t}_{\mathrm{f}}\right]\right\}$

The control system (5) is rewritten implicitly as follows:

$$
\begin{aligned}
& S^{\prime}(\mathrm{t})=\mathrm{F}(\mathrm{t}, S(\mathrm{t}), u(\mathrm{t})), \\
& S(0)=S_{0} \text { given. }
\end{aligned}
$$

with $S(\mathrm{t})=(\mathrm{x}(\mathrm{t}) \mathrm{y}(\mathrm{t}) \mathrm{z}(\mathrm{t}) \mathrm{h}(\mathrm{t}))^{T}$ is the state vector and $u(\mathrm{t})$ is the control function. The objective function (6) is implicitly defined at control $u(t)$ as follows:

$$
J(\mathrm{u})=\int_{0}^{\mathrm{t}_{\mathrm{f}}} G(t, S(t), u(t)) \mathrm{dt},
$$

Consider the problem:

$$
\begin{gathered}
\max _{u \in \mathrm{U}} \int_{0}^{\mathrm{t}_{\mathrm{f}}} G(t, S(t), u(t)) \mathrm{dt}, \\
\text { subject to } S^{\prime}(\mathrm{t})=\mathrm{F}(\mathrm{t}, S(\mathrm{t}), u(\mathrm{t})), S(0)=S_{0}, \\
0 \leq u(\mathrm{t}) \leq \lambda .
\end{gathered}
$$

The Pontryagin's maximum principle [11] provides necessary conditions for an optimal control problem. This principle converted the problem of finding a control which maximizes the objective function $J$ subject to the state ODE and initial condition to the problem of maximizing the Hamiltonian $\mathrm{H}$, pointwisely with respect to $u$ implying that it is sufficient to derive the Hamiltonian $\mathrm{H}$ instead of deriving the objective function $J$ defined in (6) in order to characterize the optimal control $u^{*}$. The Hamiltonian is defined from the formulation of the objective function as follows:

$\mathrm{H}(\mathrm{t}, \mathrm{x}, \mathrm{y}, \mathrm{z}, \mathrm{h}, u, \varphi)=\mathrm{G}(\mathrm{t}, \mathrm{S}(\mathrm{t}), u(\mathrm{t}))+\varphi \mathrm{F}(\mathrm{t}, \mathrm{S}(\mathrm{t}), u(\mathrm{t}))$

with $\varphi(\mathrm{t})=\left(\varphi_{1}(\mathrm{t}) \varphi_{2}(\mathrm{t}) \varphi_{3}(\mathrm{t}) \varphi_{4}(\mathrm{t})\right)^{T}$ is the adjoint variable vector.

Explicitly:

$$
\begin{aligned}
\mathrm{H}(\mathrm{t}, \mathrm{x}, \mathrm{y}, \mathrm{z}, \mathrm{h}, u, \varphi) & =\frac{1}{2}\left(\mathrm{x}^{2}(\mathrm{t})-\mathrm{y}^{2}(\mathrm{t})+\mathrm{z}^{2}(\mathrm{t})-\mathrm{W} u^{2}(\mathrm{t})\right) \\
& +\varphi_{1}\left[\mathrm{cy}-\mu_{2} \mathrm{x}+\frac{\mathrm{p}_{1} \mathrm{xz}}{\mathrm{g}_{1}+\mathrm{z}}+u(\mathrm{t})\right] \\
& +\varphi_{2}\left[\mathrm{r}_{2} \mathrm{y}(1-\mathrm{by})-\frac{\mathrm{axy}}{\mathrm{g}_{2}+\mathrm{y}}\right] \\
& +\varphi_{3}\left[\frac{\mathrm{p}_{2} \mathrm{xy}}{\mathrm{g}_{3}+\mathrm{y}}-\mu_{3} \mathrm{y}\right] \\
& +\varphi_{4}[u(\mathrm{t})] .
\end{aligned}
$$

The $\varphi_{\mathrm{j}}$ where $\mathrm{j}=1,2,3,4$ are our adjoint variables that determine the adjoint system which satisfies the optimality necessary conditions.

Note, that the concavity with respect to the control $u$ is correct for a maximization problem considering this second order condition:

$$
\frac{\partial^{2} H}{\partial u^{2}}=-\mathrm{W} \leq 0
$$

Maximizing the objective function $J$ is equivalent to maximize the Hamiltonian $\mathrm{H}$ which the maximum only depends on the control $u$, which implies that it is sufficient to derive $\mathrm{H}$ instead of deriving $J$ for solving the optimality system, now the initial maximization problem (7) is rewritten as follows:

$$
\mathrm{H}\left(\mathrm{t}, x^{*}, y^{*}, z^{*}, h^{*}, u^{*}, \varphi\right)=\max _{u \in \mathrm{U}} \mathrm{H}\left(\mathrm{t}, x^{*}, y^{*}, z^{*}, h^{*}, u, \varphi\right),
$$

Finally, it remains to clarify that since the Pontryagin's maximum principle converts the optimization problem into a problem of maximizing the Hamiltonian $\mathrm{H}$ (10), thus the systemic 'cost' of treatment is minimized logically including side effects and damages caused by the control as stated above in the definition of the Hamiltonian.

The existence of a solution is proved using a classical existence result by Fleming and Rishel in [12]. Thus, the following properties must be checked:

1. The class of all initial conditions with a control $u$ in the admissible control set $U$ along with each state equation being satisfied is not empty;

2. The control set $U$ is convex and closed;

3. The right-hand side of the state system is continuous, is bounded above by a sum of the bounded control and the state, and can be written as a linear function of $u$ with coefficients depending on time and the state;

4. The integrand of the objective functional $J(u)$ is concave on $\mathrm{U}$;

5. There exist constants $b_{1}, b_{2}>0$ and $\alpha>1$ such that the integrand $G(t, S(t), u(t))$ of the objective functional $J(u)$ satisfies:

$$
G(t, S(t), u(t)) \leq b_{2}-b_{1}\left(|u|^{2}\right)^{\frac{\alpha}{2}}
$$

Since the system (5) has bounded coefficients and any solutions are bounded on the finite time interval $\left[0, \mathrm{t}_{\mathrm{f}}\right]$, a result from [13] is used to obtain the existence of the solution of the system (5). The control set $\mathrm{U}$ is convex and closed by definition. The system (5) is bilinear in the control and each right hand side of this system is continuous and can be written as a linear function of $u$ with coefficients depending on time and state. Furthermore, the fact that all variables $\mathrm{x}, \mathrm{y}, \mathrm{z}, \mathrm{h}$ and $u$ are bounded on $\left[0, \mathrm{t}_{\mathrm{f}}\right]$ implies the rest of the third hypothesis. Finally, note that $(-G(t, S(t), u(t)))$ is convex in $\mathrm{U}$. Thus, it can be subsequently proved that the integrand $G(t, S(t), u(t))$ in the objective functional (6) is concave in $\mathrm{U}$.

In addition, notice that there exists a constant $\alpha>1$ and positive numbers $b_{1}$ and $b_{2}$ satisfying:

$$
\begin{aligned}
G(t, S(t), u(t)) & \leq \mathrm{x}^{2}(\mathrm{t})+\mathrm{z}^{2}(\mathrm{t})-\mathrm{W} u^{2}(\mathrm{t}) \\
& \leq b_{2}-b_{1}\left(|u|^{2}\right)^{\frac{\alpha}{2}}
\end{aligned}
$$

where $b_{2}$ depends on the upper bounds on $\mathrm{x}$ and $\mathrm{z}$ and by analogy it would be appropriate to set $b_{1}=\mathrm{W}$ and $\alpha=2$.

By applying the Pontryagin's maximum principle [11] and the existence result for the optimal control from [12] and [13], the following theorem is obtained: 


\subsubsection{Theorem}

Given optimal control $u$ and solutions $\mathrm{x}, \mathrm{y}, \mathrm{z}$ and $\mathrm{h}$ of the corresponding state system (5), there exist adjoint variables $\varphi_{1}(\mathrm{t}), \varphi_{2}(\mathrm{t}), \varphi_{3}(\mathrm{t})$ and $\varphi_{4}(\mathrm{t})$ satisfying the following equations:

$$
\begin{aligned}
\frac{\mathrm{d} \varphi_{1}}{\mathrm{dt}} & =-\left[\mathrm{x}+\varphi_{1}\left(-\mu_{2}+\frac{\mathrm{p}_{1} \mathrm{z}}{\mathrm{g}_{3}+\mathrm{z}}\right)-\frac{\varphi_{2} \mathrm{ay}}{\mathrm{g}_{2}+\mathrm{y}}+\frac{\varphi_{3} \mathrm{p}_{2} \mathrm{y}}{\mathrm{g}_{3}+\mathrm{y}}\right], \\
\frac{\mathrm{d} \varphi_{2}}{\mathrm{dt}} & =-\left[-\mathrm{y}+\varphi_{1} \mathrm{c}+\varphi_{2}\left(\mathrm{r}_{2}-2 \mathrm{r}_{2} \mathrm{by}\right)-\frac{\varphi_{2} \mathrm{~g}_{2} \mathrm{ax}}{\left(\mathrm{g}_{2}+\mathrm{y}\right)^{2}}+\frac{\varphi_{3} \mathrm{~g}_{3} \mathrm{p}_{2} \mathrm{x}}{\left(\mathrm{g}_{3}+\mathrm{y}\right)^{2}}\right], \\
\frac{\mathrm{d} \varphi_{3}}{\mathrm{dt}} & =-\left[\mathrm{z}+\frac{\varphi_{1} \mathrm{~g}_{1} \mathrm{p}_{1} \mathrm{x}}{\left(\mathrm{g}_{1}+\mathrm{z}\right)^{2}}-\varphi_{3} \mu_{3}\right], \\
\frac{\mathrm{d} \varphi_{4}}{\mathrm{dt}} & =0
\end{aligned}
$$

with transversality conditions : $\varphi_{\mathrm{i}}\left(\mathrm{t}_{\mathrm{f}}\right)=0, \mathrm{i}=1,2,3$.

Moreover, the optimal control is given by:

$$
u^{*}(\mathrm{t})=\min \left(\lambda, \max \left(0, \frac{\varphi_{1}(t)+\varphi_{4}(t)}{W}\right)\right) .
$$

\subsubsection{Proof}

Due to the concavity of integrand of $J$ with respect to $u$, a priori boundedness of the state solutions and the Lipschitz property of the state system with respect to the state variables. The existence of an optimal control has been given by [12] (see Corollary 4.1) and [13]. The adjoint equations and transversality conditions can be obtained by using Pontryagin's Maximum Principle such that:

$$
\begin{aligned}
& \frac{d \varphi_{1}}{d t}=-\frac{\partial H}{\partial x}, \\
& \frac{d \varphi_{2}}{d t}=-\frac{\partial H}{\partial y}, \\
& \frac{d \varphi_{3}}{d t}=-\frac{\partial H}{\partial z}, \\
& \frac{d \varphi_{4}}{d t}=-\frac{\partial H}{\partial h} .
\end{aligned}
$$

The optimal control $u^{*}$ can be solve from the optimality conditions:

$$
\frac{\partial H}{\partial u}=\varphi_{1}(t)+\varphi_{4}(t)-W u(t)=0
$$

By the bounds in $\mathrm{U}$ of the control, it is easy to obtain $u^{*}$ in the form of (14).

\section{NUMERICAL SIMULATION \\ 3.1 Summary of parameters and values used}

Note that it is always difficult to assign a set of general settings for patients with different cancer types and representing varying clinic cases. However, since the main purpose of this study is to use the optimal control theory for finding an optimal therapeutic strategy based on the Adoptive Cellular Immunotherapy (ACI) by introducing an isoperimetric constraint $\mathrm{C}$ that represents the treatment amount, the values of the parameters found in [7] are kept and it is stated that the stability properties of the model (5) are stored for these parameters

\subsection{Numerical method}

There are many methods and techniques of programming that can be used to solve numerically the optimal control problem
(7) and namely, to find $u^{*}$ that maximizes the objective function (6). Generally to solve the optimality system of (1), an iterative method with a Runge-Kutta fourth order scheme is used and it is known under the name of Forward Backward Sweep Method (FBSM) [14]. The principle of this method is that from an initial guess, the state system is solved forward in time and then the adjoint system is solved backward in time. All information about the convergence of this method is given in [15].

In this paper, after defining and introducing an isoperimetric constraint to the basic model (1), note that all states of the system (5) have a free end conditions except the last state $h(t)$ that both their initial and final conditions (4) are known. Our iterative method (FBSM) is limited and cannot deal with this type of problems directly. Therefore, another technique must be developed to be able to solve it numerically. Note that the adjoint system (13) satisfies the following transversality conditions:

$$
\varphi_{\mathrm{i}}\left(\mathrm{t}_{\mathrm{f}}\right)=0, \mathrm{i}=1,2,3 \text {. }
$$

where $\varphi_{4}\left(\mathrm{t}_{\mathrm{f}}\right)$ is unknown.

Firstly, suppose that $\varphi_{4}\left(\mathrm{t}_{\mathrm{f}}\right)=\theta$. Then, the optimality system is solved using the FBSM iterative method with the given initial conditions and $\vec{\varphi}\left(\mathrm{t}_{\mathrm{f}}\right)=(0,0,0, \theta)$. The iterations continue until convergence is achieved, a value of $h(t)$ at the final time $t_{f}$ is finally obtained and it is denoted by $h_{f}$ which is a function that depends on $\theta$. The main idea of this numerical solution technique is to define a new function $f(\theta)=h_{f}-C$ and to seek therefore the zeros of this function using a root-finding algorithm commonly known in numerical analysis as Secant method [14],[16]. Finally, the stored values for the variables $\mathrm{t}, \mathrm{x}, \mathrm{y}, \mathrm{z}, \mathrm{h}$ and $u$ are outputted during the last iteration, when $\mathrm{f}(\theta)$ was nearly 0 . Therefore, these are taken to be the solutions of the optimal control problem (7).

\subsection{Numerical results}

According to the definition of the control set $\mathrm{U}$, note that:

$$
0 \leq u(\mathrm{t}) \leq \lambda,
$$

Therefore the choice of the constraint C (3) is linked however, to the parameters $\lambda$ and $t_{f}$ by the relation:

$$
0 \leq C \leq \lambda \mathrm{t}_{\mathrm{f}},
$$

Note with interest that although the maximum dose that can be administered to the patient is on the order of $35 \times 10^{4}$ cell units during the 350 days of treatment, the isoperimetric constraint is set $\mathrm{C}=14 \times 10^{4}$ cell units for minimizing side effects of treatment.

Biologically, the treatment tolerance varies between patient cases. Thus, two different biological cases are studied in which the obtained results are compared using different values of the weight factor ' $\mathrm{W}$ ' for testing the effectiveness of the proposed treatment.

For numerical simulations, in addition to the optimal control $u(\mathrm{t})$ and the system states $\mathrm{x}(\mathrm{t}), \mathrm{y}(\mathrm{t}), \mathrm{z}(\mathrm{t})$ and $\mathrm{h}(\mathrm{t})$, a new function will be graphically represented:

$$
g(\mathrm{t})=\int_{0}^{\mathrm{t}_{\mathrm{f}}}(x(t)+z(t)) \mathrm{dt} .
$$


Where $g(\mathrm{t})$ represents the cumulative sum of the immune cells $x(t)$ and $z(t)$ and that will allow to have a clear idea about the total number of mobilized immune cells designed to eliminate the tumor.

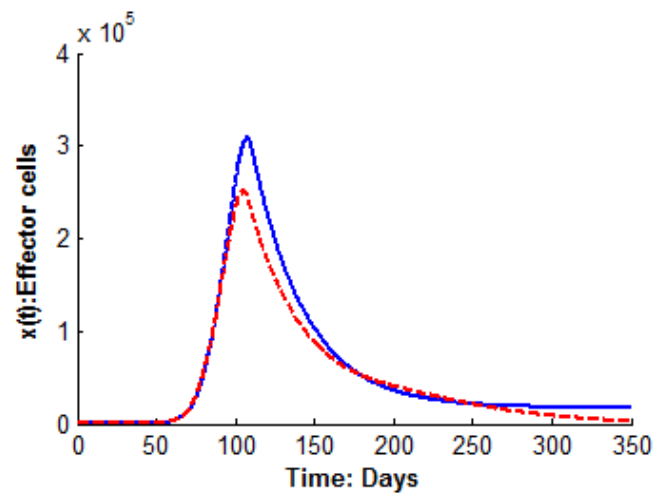

With a weight factor $W=1$. ----- With a weight factor $W=1000$.

Fig 1: The graphic of the state $x(t)$ with $(x(0)=1$ cell unit, $\mathrm{C}=14 \times 10^{4}$ cell units and $\mathrm{c}=0,015 \mathrm{day}^{-1}$ ).

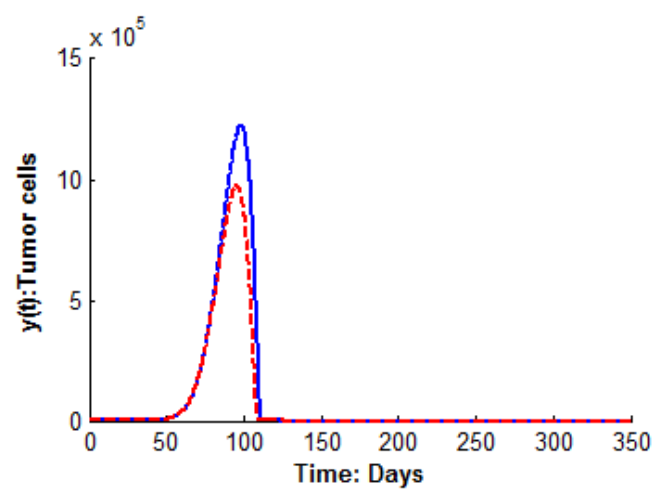

With a weight factor $\mathrm{W}=1$. ----- With a weight factor $\mathrm{W}=1000$.

Fig 2: The graphic of the state $y(t)$ with $(y(0)=1$ cell unit, $\mathrm{C}=14 \times 10^{4}$ cell units and $\left.\mathrm{c}=0,015 \mathrm{day}^{-1}\right)$.

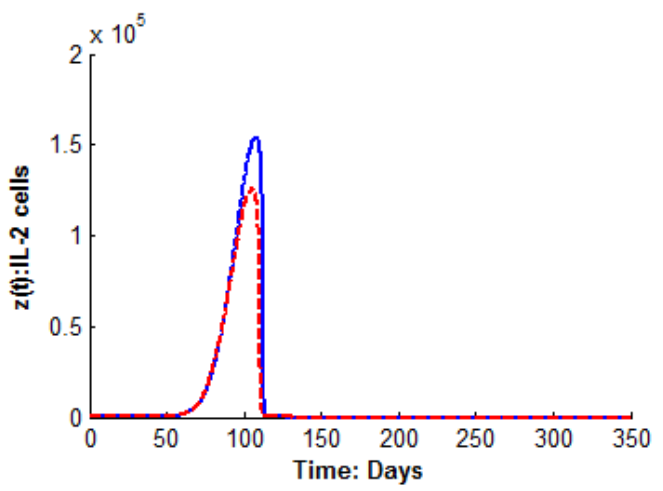

With a weight factor $\mathrm{W}=1$. ----- With a weight factor $W=1000$.

Fig 3: The graphic of the state $\mathrm{z}(\mathrm{t})$ with $(\mathrm{z}(0)=1$ cell unit, $\mathrm{C}=14 \times 10^{4}$ cell units and $\mathrm{c}=0,015 \mathrm{day}^{-1}$ ).

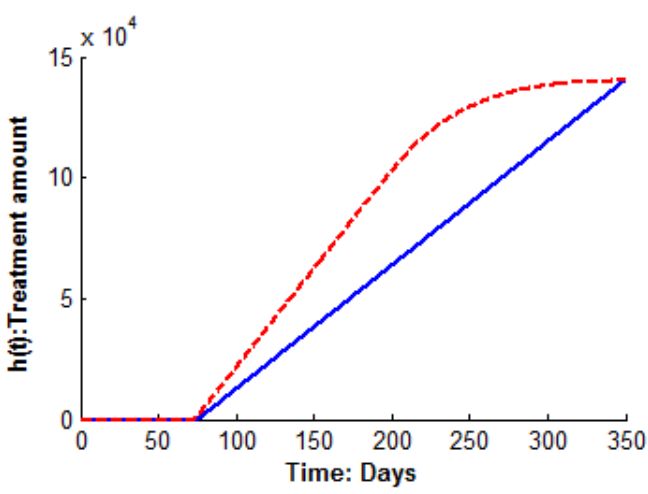

With a weight factor $\mathrm{W}=1$. With a weight factor $\mathrm{W}=1000$.

Fig 4: The graphic of the state $h(t)$ with $(h(0)=0$ cell unit, $h\left(t_{f}\right)=C=14 \times 10^{4}$ cell units and $\left.c=0,015 d a y^{-1}\right)$.

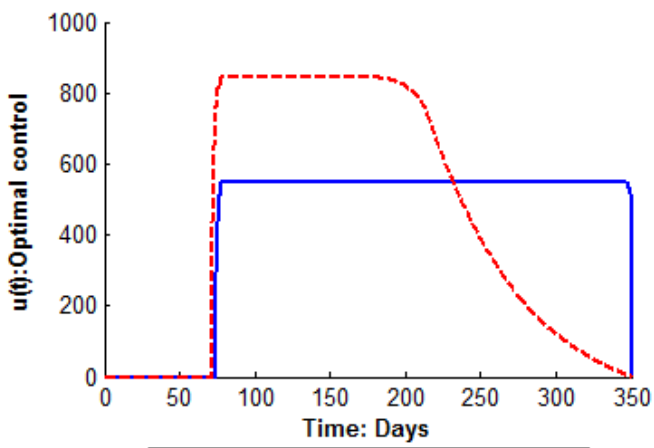

With a weight factor $\mathrm{W}=1$.

With a weight factor $\mathrm{W}=1000$.

Fig 5: The graphic of the optimal control $u^{*}$ with $(x(0)=1$ cell unit, $y(0)=1$ cell unit, $z(0)=1$ cell unit, $h(0)=0$ cell unit, $\mathrm{C}=14 \times 10^{4}$ cell units and $\mathrm{c}=0,015 \mathrm{day}^{-1}$ ).

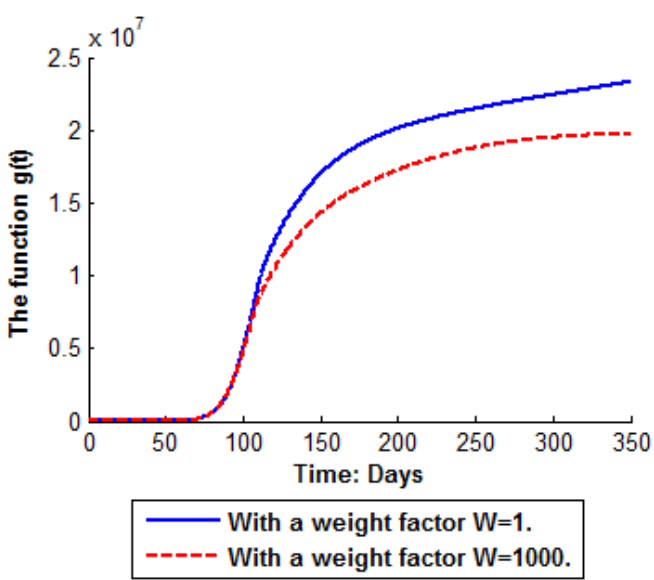

Fig 6: The graphic of the function $g(t)$ with $(x(0)=1$ cell unit, $y(0)=1$ cell unit, $z(0)=1$ cell unit, $h(0)=0$ cell unit, $\mathrm{C}=14 \times 10^{4}$ cell units and $\left.\mathrm{c}=0,015 \mathrm{day}^{-1}\right)$.

In the first biological case $(\mathrm{W}=1)$, it is noticed that the tumor occurs significantly from the 30th day of the treatment period (Fig 2) and the number of tumor cells begins to increase rapidly. At the same time, the natural immune response reacts positively increasing in a logical manner the number of effector cells and Interleukin-2 cells that acts directly on the 
tumor site (Fig 1 and Fig 3). However, tumor cells continue to proliferate in a dangerous and brutal way despite the significant mobilization of natural immune defenses of the human body.

After crossing the critical threshold of $3 \times 10^{4}$ tumor cells per day in the $75^{\text {th }}$ day, the intervention of an optimal treatment process becomes more imperative. Thereafter, a continuous growth in the treatment amount administered to the patient is observed acting for limiting the significant increase in the tumor concentration level. However from the $100^{\text {th }}$ day of the treatment period, the number of tumor cells begins to decline very rapidly and the tumor disappears completely from the $125^{\text {th }}$ day but the therapeutic process continues to operate until the end of the treatment period for coping with any sudden reappearance of the tumor.

Finally, even when the optimal dose administered to the patient is reduced swiftly during the last 10 days of the treatment period (Fig 5), the tumor does not reappear and the system remains stable. Early findings graphs (Fig 1 and Fig 3) lead to note with interest that the active immune response reacts perfectly to the dynamics of tumor cells following the same growth process.

In the second biological case, the patient's level of acceptance of the treatment is improved by increasing the weight factor's value $(\mathrm{W}=1000)$. In figure 2 , it is noticed that the tumor cells begin to proliferate sharply from the $35^{\text {th }}$ day to reach a maximum value of $9.75 \times 10^{4}$ cell units in the $95^{\text {th }}$ day. However, from the 96th day, the tumor decreases rapidly and the tumor cells disappear completely from the $128^{\text {th }}$ day. Note with interest in figure 2 that the maximum number of tumor cells is much lower $\left(9.75 \times 10^{4}\right.$ cell units $)$ compared to the first case identified in this study $\left(12.26 \times 10^{4}\right.$ cell units $)$ even with a lower number of effector cells deployed in the treatment process (Fig 6).

The therapeutic process begins early and the level of the treatment amount (Fig 5) increases rapidly from the $70^{\text {th }}$ day to limit the growth of the tumor, eventually it stabilizes at a maximum value $u=843$ units and finally decreases gradually after the disappearance of the tumor from $182^{\text {th }}$ day. Finally, it is observed that the optimal control takes into account the evolution of the tumor cells level and therefore adopts the convenient therapeutic strategy to reach the objectives set in the optimal control problem.

Early findings graphs (Fig 6) lead to note with interest that the total number of the deployed immune cells during the treatment period is around $1.975 \times 10^{7}$ cells and the active immune response including both effector cells and Interleukin-2 cells reacts positively to the tumor growth's evolution (Fig 1 and Fig 3). Note that after improving the patient's level of acceptance of the treatment, the total number of immune cells has decreased compared to the number recorded in the first biological case, limiting by the way side effects of a prolonged maximization of the immune response after complete tumor eradication.

Comparing results of this work with those established in [9], it is clear that the introduction of an isoperimetric constraint has allowed controlling and optimizing the treatment amount that can be administered to the patient during the therapy period in order to further minimize side effects and damages caused by the drugs.

In addition, the homogeneity of the objective function helped to achieve all goals set in the optimal control problem allowing to eradicate even some types of tumor cells that present a weak antigen $(\mathrm{c}=0.015)$ and which are not easily recognized by the immune response cells.

However, the use of the secant method for the numerical resolution of the optimality system has proved very useful in term of time and calculations accuracy since $\theta$ is chosen to be precisely the zero of the function $f(\theta)$ limiting thereby the multiple manual tests on the variable $\theta$ as was the case in [17]

Mathematical analysis of the stability of the model performed by Kirschner and Panetta in [7] involves that taking into account values set for the parameters 'c' $(c=0.015)$, 'W' (W $=1$ and $\mathrm{W}=1000$ ) and the maximum constant values taken by the active optimal control throughout the majority of treatment period (Fig 5), the problem is now located in a region where the equilibrium $E_{0}$ is stable and where the states $\mathrm{x}(\mathrm{t}), \mathrm{y}(\mathrm{t})$ and $\mathrm{z}(\mathrm{t})$ converge toward their respective equilibrium points, which clearly explains that the immune system succeeds in clearing the tumor confirming by the way the effectiveness of the therapeutic strategy followed and justifying the results of the numerical simulations.

\section{CONCLUSION}

In order to find an optimal treatment for cancer, a therapeutic strategy is established using the Adoptive Cellular Immunotherapy (ACI) that aims to transfer 'TIL' cells in the tumor site for strengthening the immune response to eliminate tumor cells. Firstly, a control characterizing this therapy is introduced to the basic model. Then, it is supposed that the exact treatment amount that can be administered to the cancer patient is precisely known. Thus, an isoperimetric constraint is set and the basic model is modified by adding a new state characterizing the evolution of the treatment dose while choosing an appropriate and homogeneous problem of optimal control to use properly the Pontryagin's maximum principle to finally formulate the optimal control. However, was essential to adapt the iterative FSBM method to the new problem for simulating numerically the optimality system.

Taking account of all these techniques and theoretical measurements, satisfying and beneficial numerical results were obtained and especially compatible with the model's stability analysis. The immune response has totally eliminated the tumor and established an active immune system thereby limiting the excessive use of treatment by reducing both the drug concentration and the therapy cost. One of the prospects of the future work is trying to find the optimal terminal time of treatment $t_{f}{ }^{*}$ in which the treatment cost can be further minimized while achieving all the objectives set in the optimal control problem.

\section{REFERENCES}

[1] R. Swanson, Carly Bridge, J.D. Murray, Ellsworth C Alvord Jr: Virtual and real brain tumors: using mathematical modeling to quantify glioma growth and invasion. Journal of the Neurological Sciences 216, 110 (2003).

[2] Svetlana, Bunimovich-Mendrazitsky, Eliezer Shochat, Lewi Stone: Mathematical Model of BCG Immunotherapy in Superficial Bladder Cancer. Bulletin of Mathematical Biology, DOI 10.1007/s11538-0079195-z (2007).

[3] Celestia S. Higano, Paul F. Schellhammer, Eric J. Small, Patrick A. Burch, John Nemunaitis, Lianng Yuh, Nicole Provost, Mark W. Frohlich, Integrated data from 2 randomized, double-blind, 
controlled, phase 3 trials of active cellular immunotherapy with sipuleucel-T in advanced prostate cancer. Cancer Volume 115, Issue 16, pages 36703679 (2009).

[4] Blattman JN, Greenberg PD. Cancer immunotherapy: A treatment for the masses. Science 305:200-205, (2004).

[5] L.G. de Pillis, W. Gu, A.E. Radunskaya, Mixed immunotherapy and chemotherapy of tumors: modeling, applications and biological interpretations, J. Theor. Biol. 238 (4) 841, (2006)

[6] Castiglione F, Piccoli B. Cancer immunotherapy, mathematical modeling and optimal control. J Theor Biol; 247(4):723, (2007).

[7] D. Kirschner and J. C. Panetta, Modeling Immunotherapy of the Tumor-Immune Interaction. J. Math. Biol. 37, 235-252 (1998).

[8] Cliburn Chan, Andrew George, and Jaroslav Stark, T Cell Sensitivity and Specificity, Kinetic Proofreading Revisited. Discrete and Continuous Dynamical Systems Series B, 3, 343-360 (2003).

[9] Burden, T., Ernstberger, J., Renee Fister, K. Optimal control applied to immunotherapy. Discrete Contin. Dyn. S. B 4 (1), 135146, (2004).

[10] Kamien, M. I. and N. L. Schwartz, Dynamic Optimization: The Calculus of Variations and Optimal Control in Economics and Management, 2nd ed, New York, Elsevier Science, (1991).
[11] Pontryagin, L. S., Boltyanskii, V. G., Gamkrelidze, R. V., and Mishchenko, E. F., The Mathematical Theory of Optimal Processes, Vol4, Gordon and Breach Science Publishers (1986).

[12] W. H. Fleming, R. W. Rishel. Deterministic and Stochastic Optimal Control, Springer Verlag, New York (1975).

[13] D. L. Lukes, Differential Equations: Classical To Controlled, vol.162 of Mathematics in Science and Engineering, Academic Press, New York, NY, USA (1982).

[14] S.Lenhart, T.Workman: Optimal Control Applied to Biological Models. Chapman and Hall/CRC Mathematical and Com-putational Biology Series (2007).

[15] Michael McAsey, Libin Mou, Weimin Han: Convergence of the Forward-Backward Sweep Method in Optimal Control.

[16] E. Ward Cheney, David R. Kincaid, Numerical Mathematics and Computing, Thomson, Belmont, California (2004).

[17] R. M. Neilan and S. Lenhart. An introduction to optimal control with an application in disease modeling. In modeling paradigms and analysis of disease transmission models, volume 75 of DIMACS Ser. Discrete Math. Theoret. Comput. Sci. Amer. Math. Soc., Providence, RI, (2010). 